\title{
Association between burnout and wellness culture among emergency medicine providers
}

\author{
Revathi Jyothindran, James P d'Etienne, Kevin Marcum, Amy F Ho, \\ Richard D Robinson, Aubre Tijerina, Clare Graca, Heidi C Knowles, \\ Nestor R Zenarosa, Hao Wang
}

Integrative Emergency Services, John Peter Smith Health Network, Dallas, TX, USA

Objective Burnout is a common occurrence among healthcare providers and has been associated with provider wellness culture. However, this association has not been extensively studied among emergency medicine (EM) providers. We aim to determine the association between EM provider burnout and their culture of wellness, and to elicit the independent wellness culture domains most predictive of burnout prevention.

Methods This was a multi-center observational study. We enrolled EM physicians and advanced practice providers from sixteen different emergency departments (EDs). Provider wellness culture and burnout surveys were performed. The wellness culture domains included in this study are personal/organizational value alignment, provider appreciation, leadership quality, self-controlled scheduling, peer support, and family support. Correlations between each wellness culture domain and burnout were analyzed by Pearson correlation co-efficiency, and their associations were measured by multivariate logistic regression with adjustments of other confounders.

Results A total of 242 ED provider surveys were entered for final analysis. The overall burnout rate was 54\% (130/242). Moderate correlations were found between burnout and two wellness culture domains (value alignment: $r=-0.43, P<0.001$ and provider appreciation: $r=-0.49$, $P<0.001)$. The adjusted odds ratio of provider appreciation associated with burnout was 0.44 (95\% confidence interval, $0.25-0.77 ; P=0.004$ ), adjusted odds ratio of family support was 0.67 (95\% confidence interval, $0.48-0.95 ; P=0.025$ ).

Conclusion ED providers have a relatively high burnout rate. Provider burnout might have certain associations with wellness culture domains. Provider appreciation and family support seem to play important roles in burnout protection.

Keywords Emergency medicine; Burnout, psychological; Culture

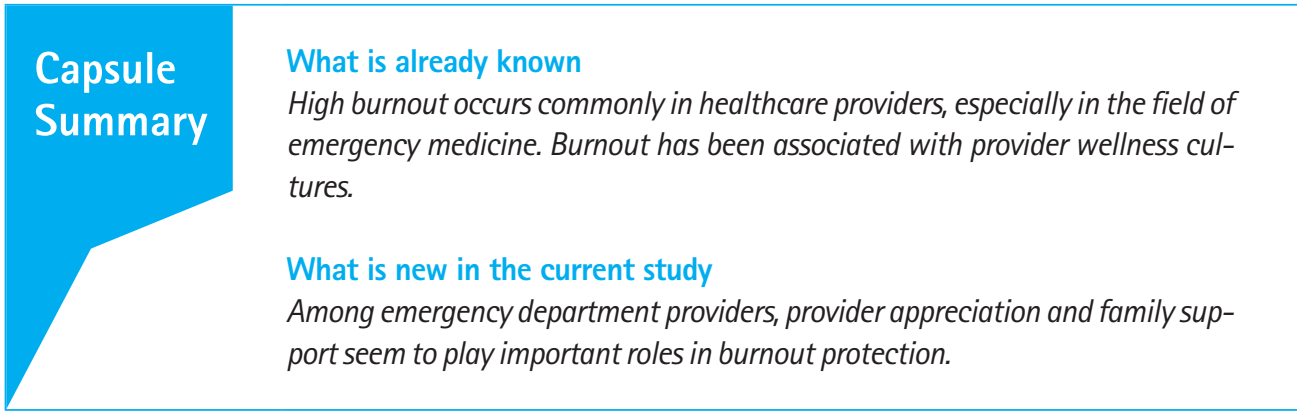

elSSN: 2383-4625

Received: 12 June 2020

Revised: 11 July 2020

Accepted: 20 July 2020

Correspondence to: Hao Wang Integrative Emergency Services, John Peter Smith Health Network, $1500 \mathrm{~S}$ Main St., Fort Worth, TX 76104, USA E-mail: hwang@ies.healthcare ORCID

https://orcid.org/0000-0002-5105-0951

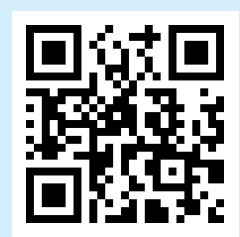

Jyothindran R, d'Etienne JP, Marcum K, Ho $A F$, Robinson RD, Tijerina A, Graca $C$, Knowles HC, Zenarosa NR, Wang $\mathrm{H}$. Association between burnout and wellness culture among emergency medicine providers. Clin Exp Emerg Med 2021;8(1):55-64. https://doi.org/10.15441/ ceem. 20.074

This is an Open Access article distributed under the terms of the Creative Commons Attribution Non-Commercial License (https:// creativecommons.org/licenses/by-nc/4.0/). 


\section{INTRODUCTION}

There is a high occurrence of burnout among healthcare providers, including nurses, physicians, pharmacists, residents, and even medical students..$^{1-4}$ High burnout among healthcare providers is associated with significant negative outcomes, including decreased job satisfaction, professional dropout, increased medical errors, increased risk of suicide, and substance abuse..$^{5-8}$ Emergency medicine (EM) providers typically work in a more stressful environment and risk higher rates of litigation as compared to other healthcare specialists. As a result, they tend to suffer a higher burnout rate and incidences of burnout related outcomes. ${ }^{6,9,10}$

Many interventions focusing on provider wellness have been reported in the literature to help prevent and ameliorate provider burnout. ${ }^{11-13}$ Increasing general self-efficacy, reducing financial debt, receiving more perceived social support, and developing a better culture of wellness are some interventions that have proven to reduce provider burnout. ${ }^{2,14,15}$ However, these claims appear to be more specialty specific, with very few applicable to EM due to limited sample size, thereby lacking generalizability and largescale external validations.

Wellness culture can be characterized by several domains. It consists of provider value alignment, perceived appreciation, leadership quality, and provider social support. Studies focused on wellness culture found that a positive culture increases provider performance, increases job security and satisfaction, and reduces provider burnout. ${ }^{16,17}$ Each individual wellness culture domain has been reported previously in the literature. However, the findings are not consistent, and each study seemed to focus on different domains. A study done by Jambrak et al. ${ }^{18}$ in Australia reported that aligning personal values with organizational values can improve job satisfaction, lower the intention to leave, and reduce burnout. However, another study done by Healthy Work Place Investigators in the US found that although clinicians with a high degree of trust are associated with high value alignment, no significant difference occurred in terms of its association with clinician burnout. ${ }^{19}$ Shanafelt et al. ${ }^{20}$ and Shanafelt and Noseworthy ${ }^{21}$ considered that the cause of burnout is not solely based upon the individual physician, rather it is a systemic issue. Therefore, executive leadership plays an important role in promoting engagement and reducing burnout. Wu et al. ${ }^{17}$ also reported that provider work-family conflicts positively affect job burnout. On the other hand, these studies focused on general practitioners and internal medicine specialists at academic centers. Therefore, it is still uncertain whether such findings can be replicated in EM with different types of EM providers practicing in different healthcare settings (i.e., academic versus community, high versus low volume emergency department [ED), etc.). In addition, most studies focused on one or two wellness culture domains, rarely considering different domains as an integrated entity. Few provided insight as to the potential interactions among different domains. We believe it is also necessary to determine whether each domain acts as an independent factor that protects against provider burnout.

A better understanding of provider wellness culture could help foster a provider wellness strategy that optimally suits provider needs, with interventions that more effectively reduce provider burnout. Reduced burnout levels would also lead to better patient-centered care. Therefore, due to the lack of sufficient data describing EM provider wellness culture in relation to burnout, we aim to conduct a multi-center observational study to determine the association between ED provider burnout and different wellness culture domains, and delineate the optimal domains from wellness culture that protect against EM provider burnout.

\section{METHODS}

\section{Study design and setting}

This was a secondary data analysis of a previous quality improvement project focusing on healthcare provider wellness. Data were collected prospectively via a Stanford Wellness online survey from January to March 2018. The survey questionnaires were provided by the Stanford WellMD Center using Qualtrics Survey software (Provo, UT, USA). We had a contractual agreement with permission to use data for secondary analysis. The survey was sent to all ED physicians and advanced practice providers (APPs). Healthcare providers were from sixteen different hospital EDs located regionally within North Texas, USA. Among the sixteen EDs, two have extremely high annual volumes (>100,000 visits/yr), in which one is an academic urban ED with an Accreditation Council for Graduate Medical Education accredited EM residency program and the other is a community urban ED. Five EDs have moderate to high annual volumes (60,000-100,000 visits/yr), while the other nine EDs have low to moderate annual volumes $(30,000-60,000$ visits/yr). Due to the nature of secondary data analysis with deidentified personal information, this study was waived for approval by the local institutional review board. Due to the nature of secondary data analysis, this study was waived for approval from local institutional review board.

\section{Study participants}

We included surveys from all qualified ED healthcare providers from sixteen different EDs who agreed to participate in this study. We excluded surveys from providers who declined to participate, 
empty surveys, incomplete surveys ( $<10 \%$ complete), and duplicate surveys.

\section{Wellness culture measurements}

In this study, wellness culture was classified and measured across the six categories listed below based on several previous studies. ${ }^{722-24}$ First, organization or personal value alignment to demonstrate the understanding of organizational value, sense of belonging or teamwork, as well as the value of personal work. Second, perceived provider appreciation to assess whether a healthcare provider's work was appreciated by their team members, supervisors, and their family. Third, leadership quality to measure overall satisfaction with a provider's supervisor in different areas (e.g., career development, empowerment, encouragement, respect, providing helpful feedback, etc.). Fourth, healthcare providers control over schedule to determine whether an individual provider is able to control their own work schedule. Fifth, peer support to measure a variety of support resources from team members (e.g., peer listening/empathy, providing solutions, lifting providers up, helping with provider's work, etc.). Sixth, family support to recognize special needs, including pregnancy needs, breast feeding/pumping needs, parental leave, and leave to care for a family member.

All of these items were graded on a 5-point Likert scale ranging from 0 (not at all, strongly disagree/dissatisfied) to 4 (completely, strongly agree/satisfied). Some items could be answered as not applicable (e.g., special needs). The overall score of each category was calculated by averaging the total item scores. If items were answered as not applicable, such items were excluded from the calculation. High scores indicated provider strong agreement or satisfaction with a given culture of wellness, whereas low scores indicated the opposite.

\section{Outcome measurements}

Healthcare provider burnout was measured as the study outcome. We used a 10-item questionnaire to measure healthcare provider burnout (Appendix 1). This burnout tool was adapted partly from a 16-point Professional Fulfillment Index covering two distinct domains (emotional exhaustion and interpersonal disengagement). ${ }^{7}$ It is also similar to the Maslach Burnout Inventory, a common burnout assessment tool used in the literature. ${ }^{25}$ Each item was scored on a 5 -point Likert scale ranging from 0 (not at all/strongly disagree) to 4 (extremely/strongly agree). The overall burnout score was calculated by averaging the total item scores.

\section{Variables}

Provider demographics included age, sex (male or female), race (White, Black or African American, Asian, or other), and ethnicity
(Hispanic/Latino or not Hispanic/Latino). Providers listed their primary practice ED. EDs were divided into three volume groups (high, > 100,000 visits/yr; moderate, 60,000-100,000 visits/yr; low, 30,000-60,000 visits/yr). In general, there was less than 2\% missing data among all variables except the "family support" domain. Since this domain mainly surveyed provider needs under special circumstances (e.g., pregnancy, breast feeding, parental leave, etc.), the number of "not applicable" answers were significantly large (Appendix 2). Therefore, multiple imputation was applied to handle these missing data.

\section{Data analysis}

Analysis of variance was used for continuous data comparisons among groups, whereas Wilcoxon rank sum test was used for categorical comparisons. We used skewness and kurtosis to determine whether each wellness culture domain score was normally distributed. $\mid$ Skewness $\mid<0.5$ was mildly to normally distributed, $1>\mid$ Skewness $\mid \geq 0.5$ was moderately skewed, and $\mid$ Skewness $\mid \geq 1$ was highly skewed. Kurtosis $>3$ was considered data less normally distributed. We used Cronbach's alpha (a) to determine internal consistency of each wellness culture domain measurement. An $a>0.8$ was considered good reliability and $a>0.7$ was considered adequate reliability. To determine the association between burnout and culture of wellness, we initially used correlation co-efficiency ( $r$ ) with $|r| \geq 0.5$ indicating strong correlation, $0.5>|r| \geq 0.3$ indicating moderate correlation, and $0.3>|r| \geq 0.1$ indicating weak correlation. Meanwhile, we initiated a univariate logistic regression to determine the association between burnout and each domain of wellness culture. Then, we fit a multivariable logistic regression model evaluating such associations with different domains of wellness culture, different roles of ED providers (physician versus APP) and different ED settings (high versus moderate versus low volume EDs) after adjustment for provider sex, age, race, and ethnicity. We determined providers of high burnout with burnout scores of more than 1 based on our providers consensus by using a modified Delphi technique along with previous similar reports. ${ }^{26-28}$ Risk predictors of burnout were determined with adjusted odds ratios with 95\% confidence intervals (Cls). All analyses were performed using Stata ver. 14.2 (StataCorp., College Station, TX, USA) with P-value $<0.05$ considered statistically significant.

\section{RESULTS}

A total of 242 surveys collected from January to March 2018 were placed in the final analysis. Table 1 shows the general characteristics of the study participants. Our study included 146 ED 
physicians and 96 APPs. Most physicians were males while most APPs were females. Most participants were White, non-Hispanic, and practicing at moderate to high volume EDs. More than half of the providers (56\% from physicians and 50\% from APPs) showed high burnout levels. However, no significant differences

Table 1. General characteristics of study participants

\begin{tabular}{|c|c|c|}
\hline Characteristics & $\begin{array}{l}\text { Physician } \\
(n=146)\end{array}$ & $\begin{array}{c}\text { APP } \\
(n=96)\end{array}$ \\
\hline \multicolumn{3}{|l|}{ Sex } \\
\hline Male & $101(69.2)$ & $34(35.4)$ \\
\hline Female & $43(29.5)$ & $62(64.6)$ \\
\hline Unknown & $2(1.4)$ & \\
\hline \multicolumn{3}{|l|}{ Age (yr) } \\
\hline$<40$ & $77(52.7)$ & $53(55.2)$ \\
\hline $40-59$ & $56(38.4)$ & $41(42.7)$ \\
\hline$\geq 60$ & $10(6.8)$ & $2(2.1)$ \\
\hline Unknown & $3(2.1)$ & \\
\hline \multicolumn{3}{|l|}{ Race } \\
\hline White & $108(74.0)$ & $75(78.1)$ \\
\hline Black or African American & $5(3.4)$ & $4(4.2)$ \\
\hline Asian & $28(19.2)$ & $13(13.5)$ \\
\hline Others ${ }^{a}$ & $5(3.4)$ & $4(4.2)$ \\
\hline \multicolumn{3}{|l|}{ Ethnicity } \\
\hline Hispanic/Latino & $4(3)$ & $4(4.2)$ \\
\hline Not Hispanic/Latino & $142(97)$ & $91(94.8)$ \\
\hline Unknown & & $1(1.0)$ \\
\hline \multicolumn{3}{|l|}{ Participant primary hospital ED size } \\
\hline Low ED annual volume $(30,000-60,000 / y r)$ & 18 (12.3) & $18(18.8)$ \\
\hline Moderate ED annual volume $(60,000-100,000 / y r)$ & $59(40.4)$ & $44(45.8)$ \\
\hline High ED annual volume (> 100,000/yr) & $66(45.2)$ & $30(31.2)$ \\
\hline Unknown & $3(2.1)$ & $4(4.2)$ \\
\hline \multicolumn{3}{|l|}{ Burnout level } \\
\hline Low burnout & $64(43.8)$ & $48(50.0)$ \\
\hline High burnout & $82(56.2)$ & $48(50.0)$ \\
\hline
\end{tabular}

Values are presented as number (\%).

APP, advanced practice provider; ED, emergency department.

a)Includes American Indian or Alaskan Native, Native Hawaiian, or Pacific Islander, or unknown. in terms of burnout levels between physicians and APPs were found (Table 1).

The six different wellness culture domains included organizational/personal value alignment (referred to as value), perceived appreciation at practice site (referred to as appreciation), leadership/experience with the supervisor (referred to as leadership), control over schedule (referred to as schedule), peer support (referred to as peer), and family support (referred to as family). Provider burnout scores and scores of their wellness culture domains are reported in Table 2. Data from wellness culture domains and burnout assessment were mildly skewed. Therefore, both mean score with standard deviation and median score with interquartile range were reported. Internal consistency was measured among different wellness culture domains using Cronbach's $a_{\text {; all }}$ showed good internal consistency (Table 2).

To determine whether burnout was associated with different ED providers and ED settings, ED providers were divided into physicians and APPs and ED settings were divided into high, moderate, and low volume groups based on annual ED volumes. No statistical significance occurred in terms of ED provider burnout levels when different ED providers and ED settings were compared $(P>0.05)$ (Table 3).

Fig. 1 shows correlations between burnout and different wellness culture domains. Moderate correlations ( $>0.3$ ) were found between burnout and four of the provider wellness culture domains (value, appreciation, schedule, and peer). High correlations $(\geq 0.5)$ were found among three wellness culture domains (value, appreciation, and leadership). Weak correlation was found between burnout and family support (Fig. 1).

To further determine the associations between burnout and provider wellness culture, both an unadjusted univariate logistic regression and adjusted multivariate logistic regression analysis were performed. All wellness culture domains showed certain associations with provider burnout by univariate regression analy-

Table 2. Descriptive analysis of healthcare provider culture of wellness and burnout

\begin{tabular}{|c|c|c|c|c|c|}
\hline Measurement & Mean $\pm S D$ & Median (IOR) & Skewness & Kurtosis & Cronbach a \\
\hline \multicolumn{6}{|l|}{ Healthcare provider culture of wellness } \\
\hline Organizational/personal values alignment & $2.42 \pm 0.82$ & $2.50(1.83-3.00)$ & -0.22 & 2.55 & 0.89 \\
\hline Provider perceived appreciation & $2.26 \pm 0.89$ & $2.20(1.60-3.00)$ & -0.14 & 2.49 & 0.89 \\
\hline Leadership quality & $3.03 \pm 0.74$ & $3.11(2.67-3.67)$ & -1.08 & 5.10 & 0.94 \\
\hline Schedule control & $1.84 \pm 0.68$ & $1.80(1.40-2.20)$ & 0.55 & 3.46 & 0.72 \\
\hline Peer support & $2.78 \pm 0.85$ & $3.00(2.25-3.25)$ & -0.71 & 3.58 & 0.91 \\
\hline Family support (original data) & $2.97 \pm 1.08$ & $3.00(2.00-4.00)$ & -0.96 & 3.22 & 0.93 \\
\hline Family support (imputed data) & $2.95 \pm 1.04$ & $3.00(2.33-4.00)$ & -0.80 & 3.33 & \\
\hline \multicolumn{6}{|l|}{ Healthcare provider burnout measurements } \\
\hline Overall burnout & $1.06 \pm 0.70$ & $1.00(0.60-1.40)$ & 0.78 & 3.67 & 0.93 \\
\hline
\end{tabular}

$S D$, standard deviation; IOR, interquartile range. 
Revathi Jyothindran, et al.

Table 3. Culture of wellness and burnout scores relative to different providers and different ED settings

\begin{tabular}{|c|c|c|c|c|c|}
\hline \multirow{2}{*}{ Measurement } & \multicolumn{2}{|c|}{ Different ED providers } & \multicolumn{3}{|c|}{ Different ED volumes } \\
\hline & Physician & APP & High & Moderate & Low \\
\hline \multicolumn{6}{|l|}{ Healthcare provider wellness culture domains } \\
\hline Organizational/personal values alignment & $2.50(1.83-3.00)$ & $2.50(1.83-3.00)$ & $2.00(1.50-2.83)$ & $2.67(2.00-3.00)$ & $2.50(1.83-3.00)$ \\
\hline Provider perceived appreciation & $2.30(1.80-3.00)$ & $2.20(1.60-3.00)$ & $2.00(1.50-3.00)$ & $2.20(1.80-3.00)$ & $2.40(1.80-3.00)$ \\
\hline Leadership quality & $3.11(2.67-3.67)$ & $3.00(2.56-3.33)$ & $2.94(2.39-3.28)$ & $3.11(2.67-3.56)$ & $3.22(2.67-3.76)$ \\
\hline Schedule control & $1.80(1.40-2.20)$ & $1.80(1.30-2.40)$ & $1.90(1.50-2.30)$ & $1.80(1.40-2.20)$ & $1.80(1.40-2.20)$ \\
\hline Peer support & $3.00(2.25-3.25)$ & $3.00(2.25-3.38)$ & $2.75(2.00-3.00)$ & $3.00(2.50-3.50)$ & $3.00(2.00-3.00)$ \\
\hline Family support (original data) & $3.00(3.00-4.00)$ & $3.00(2.00-4.00)$ & $3.00(2.00-4.00)$ & $3.00(3.00-4.00)$ & $3.00(2.16-4.00)$ \\
\hline Family support (imputed data) & $3.00(2.65-4.00)$ & $3.00(2.00-3.67)$ & $3.00(2.21-3.75)$ & $3.00(2.72-4.00)$ & $3.00(2.16-4.00)$ \\
\hline \multicolumn{6}{|l|}{ Healthcare provider burnout measurements } \\
\hline Overall burnout & $1.00(0.60-1.40)$ & $0.95(0.50-1.50)$ & $0.95(0.45-1.75)$ & $1.00(0.60-1.50)$ & $1.00(0.55-1.30)$ \\
\hline
\end{tabular}

Values are presented as median (interquartile range).

ED, emergency department; APP, advanced practice provider.

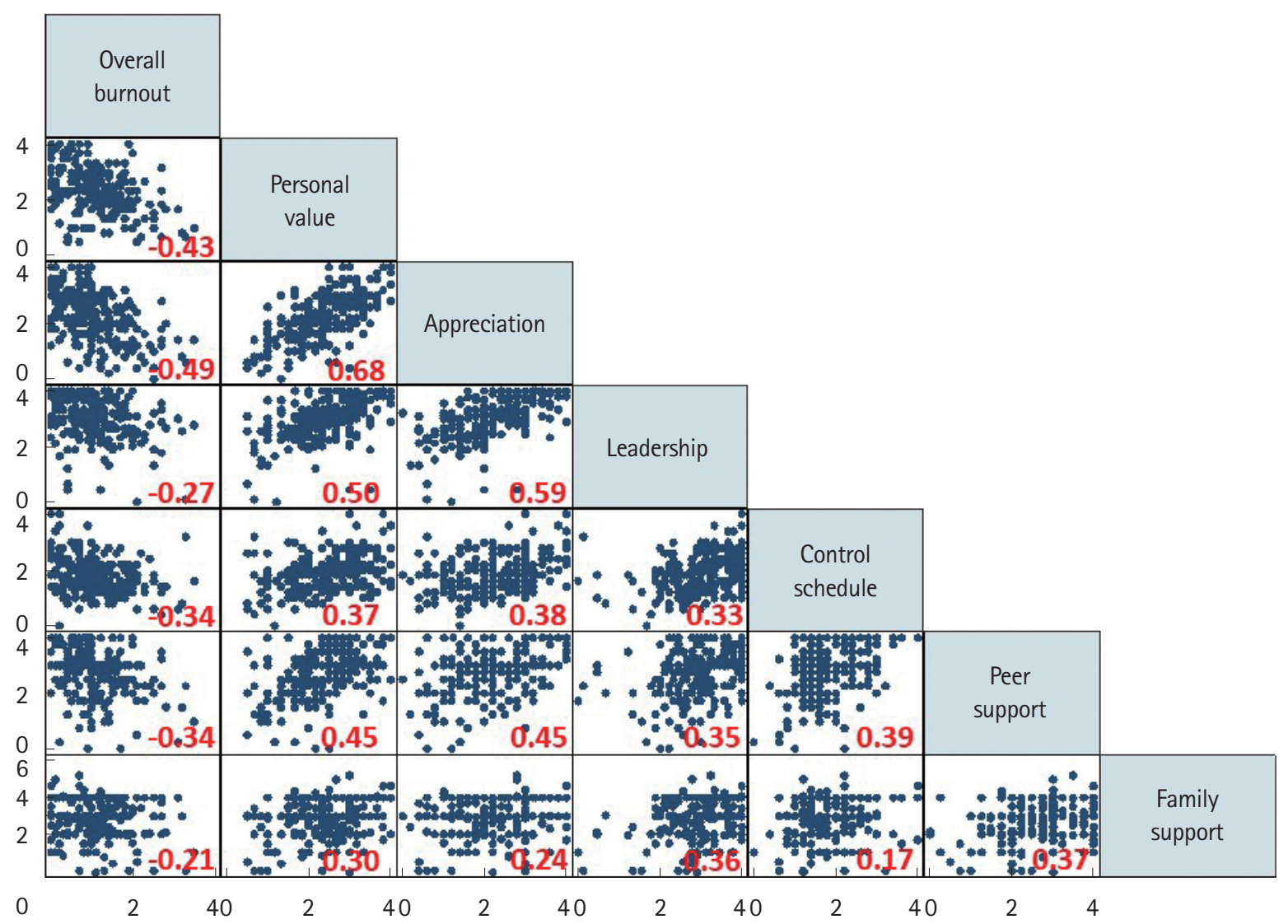

Fig. 1. Correlations between healthcare provider burnout and culture of wellness.

sis. However, when analyzed using multivariate logistic regression, only appreciation and family support domains were found to protect provider burnout significantly (Table 4). In addition, different ED providers and different ED settings seemed to have no direct association with provider burnout after all confounders were adjusted (e.g., age, sex, race, ethnicity) (Table 4).

\section{DISCUSSION}

Healthcare provider burnout has been studied frequently in recent years and is related to the quality of patient care delivered. ${ }^{29}$ Different interventions implemented to help ameliorate healthcare provider burnout have also been reported with diverse out- 
Table 4. Wellness culture domains affecting provider burnout

\begin{tabular}{|c|c|c|c|c|}
\hline & $\begin{array}{l}\text { Unadjusted odds ratio } \\
\text { (95\% confidence interval) }\end{array}$ & P-value & $\begin{array}{c}\text { Adjusted odds ratio } \\
\text { (95\% confidence interval) }\end{array}$ & P-value \\
\hline Organizational/personal value alignment & $0.41(0.29-0.59)$ & $<0.001$ & $0.75(0.42-1.32)$ & 0.317 \\
\hline Perceived appreciation at practice site & $0.35(0.25-0.51)$ & $<0.001$ & $0.44(0.25-0.77)$ & 0.004 \\
\hline Leadership/experience with the supervisor & $0.59(0.41-0.86)$ & 0.006 & $1.67(0.97-2.86)$ & 0.063 \\
\hline Control over schedule & $0.41(0.27-0.63)$ & $<0.001$ & $0.77(0.45-1.32)$ & 0.345 \\
\hline Peer support & $0.44(0.31-0.63)$ & $<0.001$ & $0.65(0.40-1.04)$ & 0.075 \\
\hline Family support (imputed) & $0.64(0.49-0.84)$ & 0.001 & $0.67(0.48-0.95)$ & 0.025 \\
\hline \multicolumn{5}{|l|}{ Provider role } \\
\hline Physician & Reference & & Reference & \\
\hline APP & $0.78(0.47-1.31)$ & 0.347 & $0.68(0.34-1.38)$ & 0.287 \\
\hline \multicolumn{5}{|l|}{ Provider at different EDs } \\
\hline High volume EDs & Reference & & Reference & \\
\hline Moderate volume EDs & $1.34(0.63-2.87)$ & 0.450 & $1.94(0.74-5.11)$ & 0.178 \\
\hline Low volume EDs & $1.04(0.48-2.24)$ & 0.915 & $1.14(0.44-2.92)$ & 0.788 \\
\hline
\end{tabular}

APP, advanced practice provider; ED, emergency department.

comes. ${ }^{18,19}$ However, EM provider wellness culture has not been widely studied and little is known of the impact of wellness culture on EM provider burnout. In order to determine the optimal intervention for EM provider burnout prevention and potential amelioration, we investigated the role of EM provider wellness culture in relation to burnout. In this study, we found that ED providers had comparable burnout levels to those of healthcare providers in prior studies. ${ }^{10,30}$ Some cultures of wellness areas showed moderate correlation with provider burnout. These areas included organizational/personal value alignment, provider appreciation, control over schedule, and peer support. However, when analyzed together, only provider appreciation and family support were found to have protective effects on EM provider burnout. Our study systematically analyzed how different wellness culture domains impacted provider burnout, identified its correlations, and determined the independent domains potentially affecting provider burnout. Such analyses have not been extensively reported in the current literature. Effectively screening for burnout and providing useful interventions for burnout prevention are very important steps in physician wellness, especially in the field of EM, a specialty with a high burnout rate. Setting up work-life balance, receiving peer and leadership support, and recognizing appreciations, which are part of the "wellness culture", have been reported separately to ameliorate burnout to certain levels from different studies. ${ }^{21,31}$ Our study provides comprehensive analyses using all the wellness culture domains to determine their association and interactions with provider burnout. Our study findings add evidence to the literature pool relative to optimizing potential effective interventions from wellness culture to help ameliorate ED provider burnout. More specifically, such findings seem to be general regardless of the different roles of
EM providers (physician versus APP) or ED settings (high versus moderate versus low volumes).

Our findings indicate that ED healthcare providers have a high burnout rate, which is consistent with other studies. ${ }^{10,30}$ Different burnout rates obtained from different burnout assessment tools could be due to different thresholds set in different studies. ${ }^{7,28}$ Our study also shows moderate correlations between burnout and most wellness culture domains. Provider appreciation is one of the wellness culture domains that significantly affect healthcare provider burnout. This has been cited in a previous report, ${ }^{32}$ yet it has not been systematically studied. Appreciation seems to bring EM providers more happiness, better job satisfaction, and results in high staff retention. ${ }^{33,34}$ This factor might effectively reduce provider anxiety and emphasize provider value at work, thus indirectly ameliorate their burnout. Family support seems to be another provider wellness culture domain that could potentially protect against provider burnout. Work-family balance has been emphasized and proven to be associated with burnout. ${ }^{17,35}$ Social support including family support has been investigated and validated to interfere with provider burnout. ${ }^{36,37}$ Female physicians burdened with high risk pregnancies and miscarriages tend to experience higher burnout rates. ${ }^{36}$ Whereas, resources at the institutional level including providing adequate breast pumping time is considered a successful strategy to support physician mothers in their careers. ${ }^{37}$ Our study extended such findings in EM. Other areas of wellness culture, though highly correlated with burnout (value, appreciation, and leadership), did not prove to independently affect provider burnout when analyzed together. This might be due to higher correlations among these factors ( $r \geq 0.5$, appreciation versus value versus leadership (Fig. 1). Others, such as control over schedule, or peer support, which could play im- 
portant roles in minimizing provider burnout, although statistically not significant, could have synergetic effects on provider appreciation (Appendix 3). However, due to a lack of strong data to support this hypothesis we are unable to explain its mechanisms thoroughly in this study. More specific studies focusing on the interactions among all these wellness culture areas are warranted in the future.

In this study, we also found provider burnout was not significantly impacted by providers working at different ED settings. In general, providers with longer-hours worked or overloaded work might suffer more stress, which could potentially result in higher burnout ${ }^{38,39}$ However, it is still unknown as to whether EM providers working in different ED settings have different levels of burnout. Our study showed no differences when comparing providers working at different ED settings. In addition, no burnout difference was found among physicians in comparison to APPs. This might be explained due to the different definitions of ED settings. In this study, we defined annual patient visits of less than 60,000 as low volume, which is different than that defined in the Emergency Department Benchmarking Alliance report. ${ }^{40}$ This study's definition is based on the fact that we have no ED within this system with an annual volume of less than 30,000 visits. Even when reclassifying all EDs into two categories (high versus moderate), our findings are still consistent with the current report (Appendix 4). Another possible reason might be due to individual provider workload although providers working at relatively low volume EDs carried similar workloads (patients per hour) when compared to providers working at relatively high volume EDs (data not shown). Therefore, we believe working at different ED settings might not be an independent critical issue affecting provider burnout.

Our study has its limitations. Since this is a retrospective observational study with secondary data analysis, patient selection bias, missing data, and inaccurate information may inevitably exist. Although this study enrolled providers from sixteen different EDs across North Texas, our sample size is small, and our findings might still be less generalizable. Additionally, provider culture of wellness could include a multitude of other domains not investigated in this study; simply analyzing six different domains might not be enough. Finally, provider burnout could be affected multifactorially, and due to limited data, we are unable to analyze all the confounders which may skew our results. Therefore, in order to accurately determine the association between EM provider burnout and culture of wellness, a large-scale multi-center prospective study is warranted for further validation.

In conclusion, ED providers have a relatively high burnout rate. Provider burnout might have certain associations with wellness culture. Provider appreciation and family support seem to play important roles in provider burnout protection.

\section{CONFLICT OF INTEREST}

No potential conflict of interest relevant to this article was reported.

\section{REFERENCES}

1. Durham ME, Bush PW, Ball AM. Evidence of burnout in health-system pharmacists. Am J Health Syst Pharm 2018; 75:S93-100.

2. Royce TJ, Davenport KT, Dahle JM. A burnout reduction and wellness strategy: personal financial health for the medical trainee and early career radiation oncologist. Pract Radiat Oncol 2019;9:231-8.

3. Romani M, Ashkar K. Burnout among physicians. Libyan J Med 2014;9:23556.

4. Dyrbye L, Shanafelt T. A narrative review on burnout experienced by medical students and residents. Med Educ 2016;50: 132-49.

5. Hirsch 0, Adarkwah CC. The issue of burnout and work satisfaction in younger GPs-A cluster analysis utilizing the HaMEdSi study. Int J Environ Res Public Health 2018;15:2190.

6. Stehman CR, Testo Z, Gershaw RS, Kellogg AR. Burnout, drop out, suicide: physician loss in emergency medicine, part I. West J Emerg Med 2019;20:485-94.

7. Trockel $M$, Bohman $B$, Lesure $E$, et al. A brief instrument to assess both burnout and professional fulfillment in physicians: reliability and validity, including correlation with self-reported medical errors, in a sample of resident and practicing physicians. Acad Psychiatry 2018;42:11-24.

8. Jackson ER, Shanafelt TD, Hasan O, Satele DV, Dyrbye LN. Burnout and alcohol abuse/dependence among U.S. medical students. Acad Med 2016;91:1251-6.

9. Shanafelt TD, Boone $S$, Tan $L$, et al. Burnout and satisfaction with work-life balance among US physicians relative to the general US population. Arch Intern Med 2012;172:1377-85.

10. Goldberg R, Boss RW, Chan L, et al. Burnout and its correlates in emergency physicians: four years' experience with a wellness booth. Acad Emerg Med 1996;3:1156-64.

11. West $C P$, Dyrbye $L N$, Shanafelt TD. Physician burnout: contributors, consequences and solutions. J Intern Med 2018;283: 516-29.

12. Panagioti $M$, Geraghty $K$, Johnson J. How to prevent burnout in cardiologists? A review of the current evidence, gaps, and 
future directions. Trends Cardiovasc Med 2018;28:1-7.

13. Yester M. Work-life balance, burnout, and physician wellness. Health Care Manag (Frederick) 2019;38:239-46.

14. Molero Jurado MDM, Perez-Fuentes MDC, Gazquez Linares JJG, Simon Marquez MDM, Martos Martinez A. Burnout Risk and Protection Factors in Certified Nursing Aides. Int J Environ Res Public Health 2018;15:1116.

15. Shapiro DE, Duquette C, Abbott LM, Babineau T, Pearl A, Haidet P. Beyond burnout: a physician wellness hierarchy designed to prioritize interventions at the systems level. Am J Med 2019;132:556-63.

16. Martin M. Physician well-being: physician burnout. FP Essent 2018;471:11-5.

17. Wu G, Wu Y, Li H, Dan C. Job burnout, work-family conflict and project performance for construction professionals: the moderating role of organizational support. Int J Environ Res Public Health 2018;15:2869.

18. Jambrak J, Deane FP, Williams V. Value motivations predict burnout and intentions to leave among mental health professionals. J Ment Health 2014;23:120-4.

19. Linzer M, Poplau S, Prasad K, et al. Characteristics of health care organizations associated with clinician trust: results from the healthy work place study. JAMA Netw Open 2019;2:e196201.

20. Shanafelt TD, Gorringe G, Menaker R, et al. Impact of organizational leadership on physician burnout and satisfaction. Mayo Clin Proc 2015;90:432-40.

21. Shanafelt TD, Noseworthy JH. Executive leadership and physician well-being: nine organizational strategies to promote engagement and reduce burnout. Mayo Clin Proc 2017;92: 129-46.

22. Trockel M. Professional fulfillment model [Internet]. Redwood City, CA: Stanford University Human Resources; 2016 [cited 2020 Jun 12]. Available from: https://wellness.healthysteps4u.org/professional-fulfillment-model/

23. Sexton JB, Schwartz SP, Chadwick WA, et al. The associations between work-life balance behaviours, teamwork climate and safety climate: cross-sectional survey introducing the worklife climate scale, psychometric properties, benchmarking data and future directions. BMJ Qual Saf 2017;26:632-40.

24. Aronsson $G$, Theorell $T$, Grape $T$, et al. A systematic review including meta-analysis of work environment and burnout symptoms. BMC Public Health 2017;17:264.

25. Williamson K, Lank PM, Cheema N, Hartman N, Lovell EO; Emergency Medicine Education Research Alliance (EMERA). Comparing the Maslach Burnout Inventory to other well-being instruments in emergency medicine residents. J Grad Med Educ 2018;10:532-6.
26. Boulkedid R, Abdoul H, Loustau M, Sibony O, Alberti C. Using and reporting the Delphi method for selecting healthcare quality indicators: a systematic review. PLoS One 2011;6:e20476.

27. McMurray JE, Linzer M, Konrad TR, Douglas J, Shugerman R, Nelson $K$. The work lives of women physicians results from the physician work life study. The SGIM Career Satisfaction Study Group. J Gen Intern Med 2000;15:372-80.

28. Dolan ED, Mohr D, Lempa $M$, et al. Using a single item to measure burnout in primary care staff: a psychometric evaluation. J Gen Intern Med 2015;30:582-7.

29. Panagioti M, Geraghty K, Johnson J, et al. Association between physician burnout and patient safety, professionalism, and patient satisfaction: a systematic review and meta-analysis. JAMA Intern Med 2018;178:1317-30.

30. Abdo SA, El-Sallamy RM, El-Sherbiny AA, Kabbash IA. Burnout among physicians and nursing staff working in the emergency hospital of Tanta University, Egypt. East Mediterr Health J 2016;21:906-15.

31. Shanafelt TD, West CP, Sinsky $C$, et al. Changes in burnout and satisfaction with work-life integration in physicians and the general US working population between 2011 and 2017. Mayo Clin Proc 2019;94:1681-94.

32. Khoo EJ, Aldubai S, Ganasegeran K, Lee BX, Zakaria NA, Tan KK. Emotional exhaustion is associated with work related stressors: a cross-sectional multicenter study in Malaysian public hospitals. Arch Argent Pediatr 2017;115:212-9.

33. Deriba BK, Sinke SO, Ereso BM, Badacho AS. Health professionals' job satisfaction and associated factors at public health centers in West Ethiopia. Hum Resour Health 2017;15:36.

34. Maurits EE, de Veer AJ, van der Hoek LS, Francke AL. Factors associated with the self-perceived ability of nursing staff to remain working until retirement: a questionnaire survey. BMC Health Serv Res 2015;15:356.

35. Pecino V, Manas-Rodriguez MA, Diaz-Funez PA, Aguilar-Parra JM, Padilla-Gongora D, Lopez-Liria R. Interpersonal justice climate, extra-role performance and work family balance: A multilevel mediation model of employee well-being. PLoS One 2018;13:e0207458.

36. Gyorffy Z, Dweik D, Girasek E. Reproductive health and burnout among female physicians: nationwide, representative study from Hungary. BMC Womens Health 2014;14:121.

37. Juengst SB, Royston A, Huang I, Wright B. Family leave and return-to-work experiences of physician mothers. JAMA Netw Open 2019;2:e1913054.

38. Allegra CJ, Hall R, Yothers G. Prevalence of burnout in the u.s. Oncology community: results of a 2003 survey. J Oncol Pract 2005;1:140-7. 
Revathi Jyothindran, et al.

39. Busireddy KR, Miller JA, Ellison K, Ren V, Qayyum R, Panda M. Efficacy of interventions to reduce resident physician burnout: a systematic review. J Grad Med Educ 2017;9:294-301.
40. Welch S, Augustine J, Camargo CA Jr, Reese C. Emergency department performance measures and benchmarking summit. Acad Emerg Med 2006;13:1074-80. 
Appendix 1. Burnout questionnaire

\begin{tabular}{|c|c|c|c|c|c|}
\hline Questionnaires & 0 & 1 & 2 & 3 & 4 \\
\hline To what degree have you experienced the following? & Not at all & Very little & Moderately & A lot & Extremely \\
\hline
\end{tabular}

Q1: During the past two weeks I have felt a sense of dread when I think about work I have to do

02: During the past two weeks I have felt physically exhausted at work

Q3: During the past two weeks I have felt lacking in enthusiasm at work

04: During the past two weeks I have felt emotionally exhausted at work

05: During the past two weeks my job has contributed to me feeling: less empathetic with my patients

Q6: During the past two weeks my job has contributed to me feeling: less empathetic with my colleagues

Q7: During the past two weeks my job has contributed to me feeling: less sensitive to others' feelings/emotions

Q8: During the past two weeks my job has contributed to me feeling: less interested in talking with my patients

09: During the past two weeks my job has contributed to me feeling: less connected with my patients

Q10: During the past two weeks my job has contributed to me feeling: less connected with my colleagues

Appendix 2. Study missing data

\begin{tabular}{lc}
\hline & Missing data \\
\hline Age & $3(1.2)$ \\
Rex & $2(0.8)$ \\
Race & $4(1.8)$ \\
Ethnicity & $1(0.4)$ \\
Burnout & $0(0)$ \\
Value alignment & $1(0.4)$ \\
Appreciation to providers & $0(0)$ \\
Leadership quality & $0(0)$ \\
Control to schedule & $0(0)$ \\
Peer support & $1(0.4)$ \\
Family need support & $80(33.1)$ \\
\hline
\end{tabular}

Values are presented as number (\%).
Appendix 4. Culture of wellness and burnout scores in related to different ED settings

\begin{tabular}{lccc}
\hline \multirow{2}{*}{ Measurement } & \multicolumn{2}{c}{ Different ED settings } & \\
\cline { 2 - 3 } & $\begin{array}{c}\text { High volume } \\
(>100,000 \text { visits/yr })\end{array}$ & $\begin{array}{c}\text { Non-high volume } \\
(\leq 100,000 \text { visits/yr })\end{array}$ & \\
\hline Overall burnout & & & \\
Mean (SD) & $1.17(0.93)$ & $1.03(0.65)$ & 0.25 \\
Median (IOR) & $0.95(0.45-1.75)$ & $1.00(0.60-1.40)$ & 0.73 \\
\hline
\end{tabular}

ED, emergency department; SD, standard deviation; IQR, interquartile range.

Appendix 3. Potential synergetic effects of wellness culture domains affecting provider burnout

\begin{tabular}{lcc}
\hline & Adjusted odds ratio (95\% confidence Interval) \\
\hline High appreciation & $0.33(0.14-0.77)$ \\
High appreciation + high peer support & $0.75(0.13-4.40)$ \\
High appreciation + high peer support + better control over schedule & $0.26(0.02-2.74)$ \\
\hline
\end{tabular}

